Supplement of Earth Syst. Sci. Data, 13, 809-825, 2021

https://doi.org/10.5194/essd-13-809-2021-supplement

(c) Author(s) 2021. This work is distributed under

the Creative Commons Attribution 4.0 License.

(c) (1)

乌. Earth System

¿̛̀ Science

气Data

Supplement of

\title{
Homogenization of the historical series from the Coimbra Magnetic Ob- servatory, Portugal
}

\author{
Anna L. Morozova et al. \\ Correspondence to: Anna L. Morozova (annamorozovauc@gmail.com)
}

The copyright of individual parts of the supplement might differ from the CC BY 4.0 License. 
Table S1 contains COI metadata (dates of the instrument replacements, relocations, changes in the measurement/calculation procedures etc.) for the D geomagnetic field element.

Table S2 gives information about sets of geomagnetic field elements measured at COI at different epochs between 1866 and 2015.

Table S3 lists instruments used for the absolute measurements of geomagnetic field elements at COI at different epochs between 1866 and 2015

Table S4 contains a list of European geomagnetic observatories whose data were used as reference series for the COI $\mathrm{H}$, I and $\mathrm{Z}$ series.

Figure S1 shows variations of the COI SV series.

Figure 2 shows differences between COV-OBS model estimations calculated with all available stations data and without COI data.

Figures S3-S9 show homogeneity tests statistics for the original and corrected COI H (S3-S5), I (S6-S7) and Z (S8-S9) series.

Figure S10 shows time derivative of the COI H, I and Z series on the month-to-month time scale.

Figure S11 shows $\Delta \mathrm{H}$ series for COI and several reference observatories (SFS, PRA, PER, OSL, PSM and GRW).

Figure S12 allows to compare COI Z variations for the 1990-2006 period with monthly mean COI temperature series. 
Table S1. Metadata for the D data set: dates of the instrument replacements, relocations, changes in the measurement/calculation procedures etc. (adapted from Morozova et al., 2014).

\begin{tabular}{|c|c|c|}
\hline & date & metadata \\
\hline \multirow{4}{*}{ 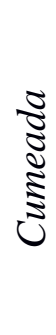 } & 1875 July (?) & $\begin{array}{l}\text { new method to calculate D was applied; new azimuth mark was } \\
\text { calculated }\end{array}$ \\
\hline & 1878 January & Unifilar of Elliot was installed \\
\hline & 1919 January & new procedure to compute the data \\
\hline & 1930 January & tram line installation \\
\hline \multirow{9}{*}{ 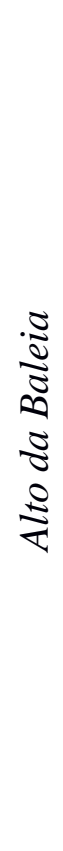 } & 1932 January & relocation to Alto da Baleia \\
\hline & $\begin{array}{l}1948 \text { October - } \\
1951 \text { March }\end{array}$ & no measurements, data in the annual books were interpolated \\
\hline & 1951 October & $\begin{array}{l}\text { reinstallation of variographs; new routines for absolute } \\
\text { measurements }\end{array}$ \\
\hline & 1955 July & $\begin{array}{l}\text { installation of a new declinometer (Askania) - after comparison } \\
\text { with former Elliot no significant differences were found }\end{array}$ \\
\hline & 1989 May & small instrumental problems and construction in the nearby area \\
\hline & 1990 June-July & small instrumental problems and construction in the nearby area \\
\hline & 1994 May & change of the suspension wire \\
\hline & 2003 March & change of the suspension wire; correction already applied \\
\hline & 2006 January & $\begin{array}{l}\text { new instrument was installed in } 2006 / 06 \text {; the correction of }-6 \text { ' was } \\
\text { applied to the data starting in } 2006 / 01\end{array}$ \\
\hline
\end{tabular}


Table S2. Measured geomagnetic field elements at COI between 1866 and 2015.

\begin{tabular}{|c|c|c|c|c|}
\hline Component & \multicolumn{2}{|l|}{ Cumeada site } & \multicolumn{2}{|c|}{ Alto da Baleia site } \\
\hline $\mathrm{D}$ & \multicolumn{2}{|l|}{$1866-1932$} & \multicolumn{2}{|c|}{$\begin{array}{l}1932-1951 \\
1951-2015\end{array}$} \\
\hline $\mathrm{H}$ & \multicolumn{2}{|l|}{$1866-1932$} & \multicolumn{2}{|l|}{$\begin{array}{l}1932-1941 \\
1951-2006\end{array}$} \\
\hline I & \multicolumn{2}{|l|}{$1866-1932$} & \multicolumn{2}{|l|}{$\begin{array}{l}1932-1940 \\
2007-2015\end{array}$} \\
\hline $\mathrm{Z}$ & \multicolumn{2}{|l|}{-} & \multicolumn{2}{|l|}{$1951-2006$} \\
\hline $\mathrm{F}$ & \multicolumn{2}{|l|}{-} & \multicolumn{2}{|l|}{$2007-2015$} \\
\hline \multicolumn{5}{|l|}{ Measured elements } \\
\hline & $1864-1931$ & $1932-1941$ & $1951-2006$ & $2007-2015$ \\
\hline $\begin{array}{l}\text { Absolute measurements } \\
\text { (baselines) }\end{array}$ & HDI & HDI & $\mathrm{HDZ}$ & DIF \\
\hline $\begin{array}{l}\text { Relative measurements } \\
\text { (magnetograms) }\end{array}$ & HDZ & HDZ & $\mathrm{HDZ}$ & $\mathrm{HDZ}$ \\
\hline
\end{tabular}


Table S3. Instruments used at COI for the absolute measurements of the geomagnetic field elements.

\begin{tabular}{|c|c|c|}
\hline Element & Instrument & Time interval. \\
\hline \multirow{4}{*}{$\mathrm{D}$} & Gibson unifilar magnetometer & JUL 1867 - DEC 1877 \\
\hline & Elliott \& Bros. unifilar magnetometer & $\begin{array}{l}\text { JAN } 1878 \text { to } 1948 \\
1951-1955\end{array}$ \\
\hline & Askania declinometer & JUL 1955 - DEC 2005 \\
\hline & $\begin{array}{l}\text { DI-flux: fluxgate Mag-01H magnetometer (Bartington) \& } \\
\text { universal theodolite YOM MG } 2 \mathrm{Kp}\end{array}$ & JAN 2006 - present time \\
\hline \multirow{7}{*}{$\mathrm{H}$} & Gibson unifilar magnetometer & JUN 1866 - DEC1877 \\
\hline & Elliott \& Bros. unifilar magnetometer & $\begin{array}{l}\text { JAN } 1878 \text { to } 1948 \\
\text { OCT } 1951-\text { DEC } 1951\end{array}$ \\
\hline & quartz horizontal magnetometers (QHM): & $1951-2006$ \\
\hline & QHM nos. 190 and 191 & JAN - OCT 1952 \\
\hline & QHM nos. 220 and 221 & NOV - DEC 1952 \\
\hline & QHM no. 220 & JAN 1953 - JUN 1955 \\
\hline & QHM no. 307 & JUL 1955 -MAY 2006 \\
\hline \multirow{4}{*}{ I } & inclinometer of Barrow & JUN 1866 - AUG1876 \\
\hline & John Dover inclinometer & SEP 1876 - SEP 1935 \\
\hline & Sartorius Earth inductor & OCT 1935 - DEC 1938 \\
\hline & DI-flux (see D element) & JAN 2006 - present time \\
\hline \multirow{4}{*}{$\mathrm{Z}$} & zero balance magnetometers (BMZ): & $1951-2006$ \\
\hline & BMZ no. 69 & OCT 1951 - JAN 1952 \\
\hline & BMZ no. 80 & JAN 1953 - DEC 1976 \\
\hline & BMZ no. 130 & JAN 1977 - MAY 2006 \\
\hline \multirow{3}{*}{$\mathrm{F}$} & proton magnetometer Geometrics G-856 & JUN 2006 - APR 2007 \\
\hline & proton magnetometer Geometrics G- 856 & MAY 2007 - JUN 2007 \\
\hline & Overhauser GSM-90F1 magnetometer (GEM systems & JUL 2007 - present time \\
\hline
\end{tabular}


Table S4. A list of European observatories (EOs) used to obtain the reference series for H, I and Z. Some stations suffered changes in the instruments and/or were re-located.

\begin{tabular}{|c|c|c|c|c|c|c|c|}
\hline & name & code & country & lat. & long. & period & $\begin{array}{c}\text { Used as reference } \\
\text { series for... }\end{array}$ \\
\hline \multirow[b]{3}{*}{1} & $\begin{array}{c}\text { Parc Saint-Maur } \\
\text { (Paris) }\end{array}$ & PSM & & $48^{\circ} 49^{\prime}$ & $2^{\circ} 30^{\prime}$ & $1883-1900$ & \multirow[b]{3}{*}{$\mathrm{H}, \mathrm{I}, \mathrm{Z}$} \\
\hline & Val Joyeux & VLJ & France & $48^{\circ} 49^{\prime}$ & $2^{\circ} 01^{\prime}$ & & \\
\hline & $\begin{array}{c}\text { Chambon-la- } \\
\text { Foret }\end{array}$ & CLF & & $48^{\circ} 01^{\prime}$ & $2^{\circ} 16^{\prime}$ & $\begin{array}{l}\text { 1936-1956; } \\
\text { 1957-1967; } \\
\text { 1968-1982; } \\
\text { 1983-2011 }\end{array}$ & \\
\hline 2 & Nantes & NTS & France & $47^{\circ} 15^{\prime}$ & $358^{\circ} 26^{\prime}$ & $1923-1958$ & $\mathrm{H}, \mathrm{I}$ \\
\hline 3 & Perpignan & PER & France & $42^{\circ} 42^{\prime}$ & $02^{\circ} 53^{\prime}$ & $\begin{array}{l}\text { 1886-1901; } \\
1907-1910 \\
\end{array}$ & $\mathrm{H}, \mathrm{I}$ \\
\hline \multirow[b]{2}{*}{4} & Toledo & TOL & \multirow[b]{2}{*}{ Spain } & $39^{\circ} 53^{\prime}$ & $355^{\circ} 27^{\prime}$ & $1947-1981$ & \multirow[b]{2}{*}{$\mathrm{H}, \mathrm{Z}$} \\
\hline & $\begin{array}{c}\text { San Pablo } \\
\text { de los Montes }\end{array}$ & SPT & & $39^{\circ} 33^{\prime}$ & $355^{\circ} 39^{\prime}$ & $1981-2011$ & \\
\hline 5 & Ebro (Tortosa) & EBR & Spain & $40^{\circ} 49^{\prime}$ & $0^{\circ} 30^{\prime}$ & $\begin{array}{l}\text { 1909-1937; } \\
\text { 1943-1983; } \\
\text { 1995-2009 }\end{array}$ & $\mathrm{H}, \mathrm{I}, \mathrm{Z}$ \\
\hline 6 & Logrono & LGR & Spain & $42^{\circ} 27^{\prime}$ & $357^{\circ} 30^{\prime}$ & $1957-1976$ & $\mathrm{H}, \mathrm{Z}$ \\
\hline \multirow[t]{2}{*}{7} & San Fernando & SFS & Spain & $36^{\circ} 28^{\prime}$ & $353^{\circ} 48^{\prime}$ & $\begin{array}{l}\text { 1880-1963; } \\
\text { 1964-1969; } \\
1970-1979\end{array}$ & \multirow[t]{2}{*}{$\mathrm{H}, \mathrm{I}, \mathrm{Z}$} \\
\hline & San Fernando & SFS2 & & $36^{\circ} 30^{\prime}$ & $353^{\circ} 53^{\prime}$ & $1991-2005$ & \\
\hline 8 & Oslo & OSL & Norway & $59^{\circ} 55^{\prime}$ & $10^{\circ} 43^{\prime}$ & $1843-1930$ & $\mathrm{H}, \mathrm{I}$ \\
\hline 9 & Prague & PRA & $\begin{array}{c}\text { Czech } \\
\text { Republic }\end{array}$ & $50^{\circ} 05^{\prime}$ & $14^{\circ} 25^{\prime}$ & $1830-1926$ & $\mathrm{H}$ \\
\hline 10 & Eskdalemuir & ESK & UK & $55^{\circ} 19^{\prime}$ & $356^{\circ} 48^{\prime}$ & $1908-2011$ & $\mathrm{H}, \mathrm{I}, \mathrm{Z}$ \\
\hline 11 & Greenwich & GRW & UK & $51^{\circ} 29^{\prime}$ & $0^{\circ} 0^{\prime}$ & $1840-1925$ & $\mathrm{H}$ \\
\hline 12 & Munich & $\mathrm{MNH}$ & Germany & $48^{\circ} 09^{\prime}$ & $11^{\circ} 37^{\prime}$ & $\begin{array}{l}\text { 1841-1886; } \\
1899-1926 \\
\end{array}$ & $\mathrm{H}, \mathrm{I}$ \\
\hline 13 & Potsdam & POT & Germany & $52^{\circ} 23^{\prime}$ & $13^{\circ} 4^{\prime}$ & $1890-2011$ & $\mathrm{H}, \mathrm{I}, \mathrm{Z}$ \\
\hline 14 & Lisbon & LIS & Portugal & $38^{\circ} 43^{\prime}$ & $350^{\circ} 51^{\prime}$ & $1858-1900$ & $\mathrm{H}, \mathrm{I}$ \\
\hline 15 & L'Aquila & AQU & Italy & $42^{\circ} 23^{\prime}$ & $13^{\circ} 19^{\prime}$ & 1960-2009 & $\mathrm{H}, \mathrm{Z}$ \\
\hline
\end{tabular}



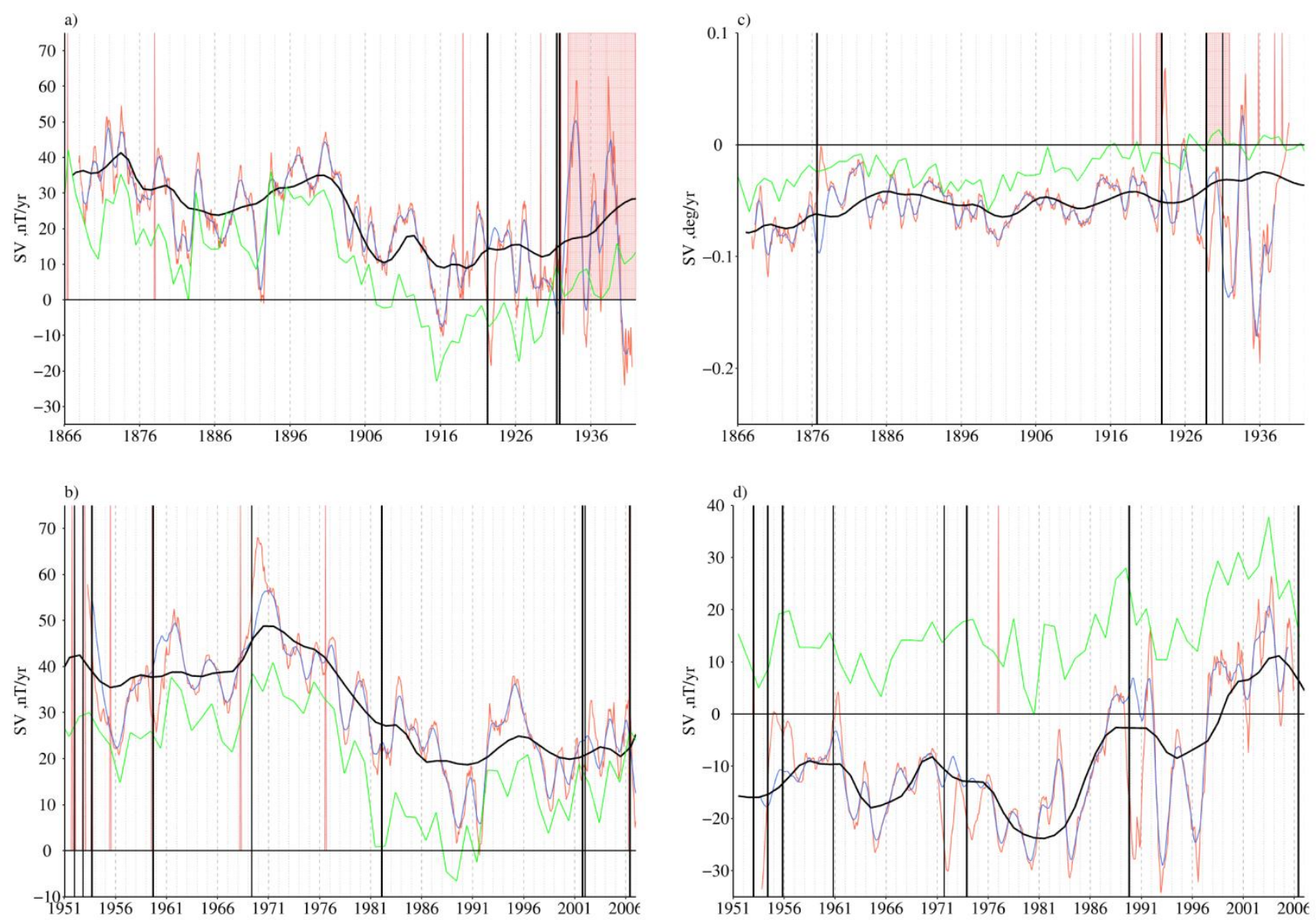

Figure S1. Original (red lines) and corrected (blue lines) COI SV H (a and b), I (c) and Z (d) series smoothed by the moving averaging with 12-month long window, SV from the corresponding COVOBS model (black lines) and SV of the reference series from the EO observatories (green lines). Pink vertical lines/rectangles mark possible dates of $\mathrm{HB}$, black vertical lines mark dates of corrections. 

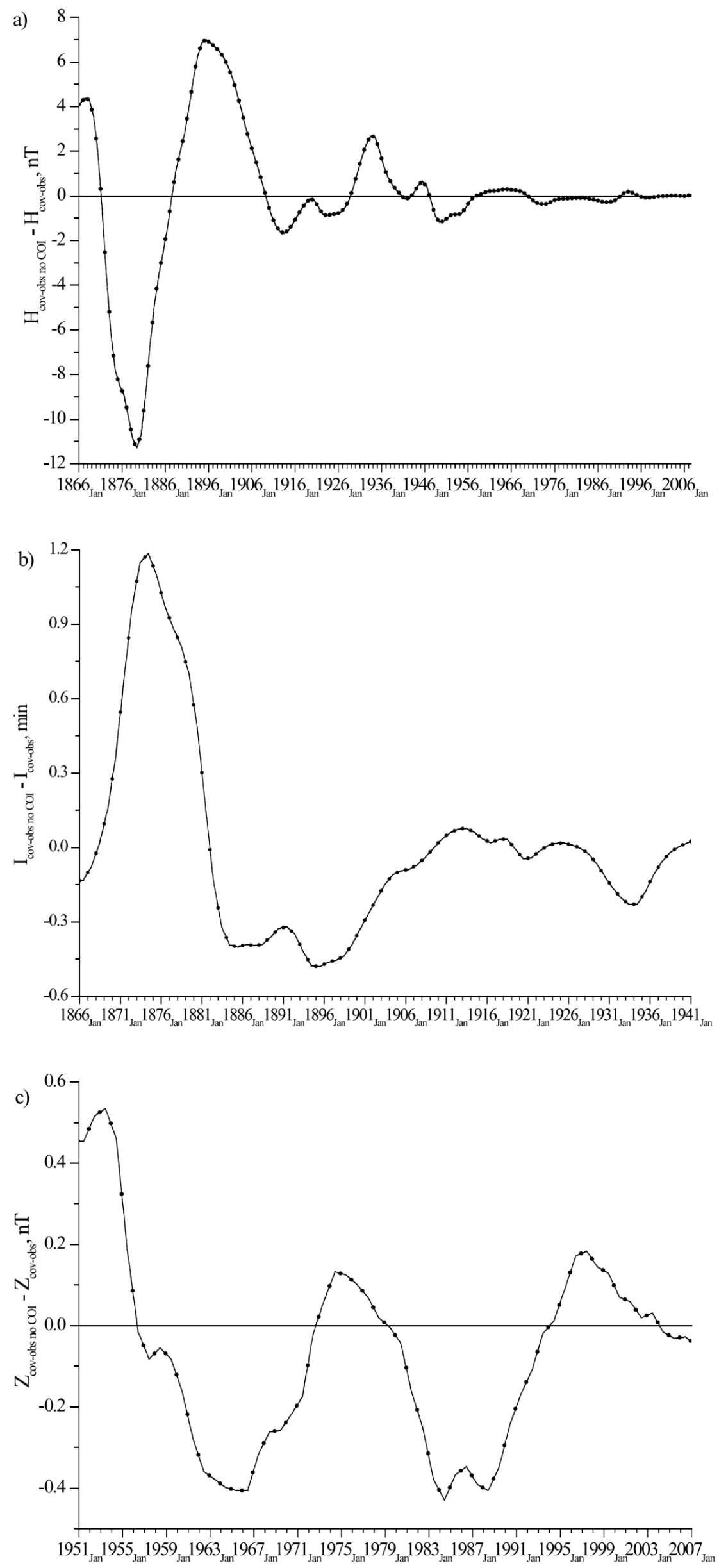

Figure S2. Differences between two models: COV-OBS and COV-OBS without COI: H (a), I (b), and $\mathrm{Z}$ (c) components. 

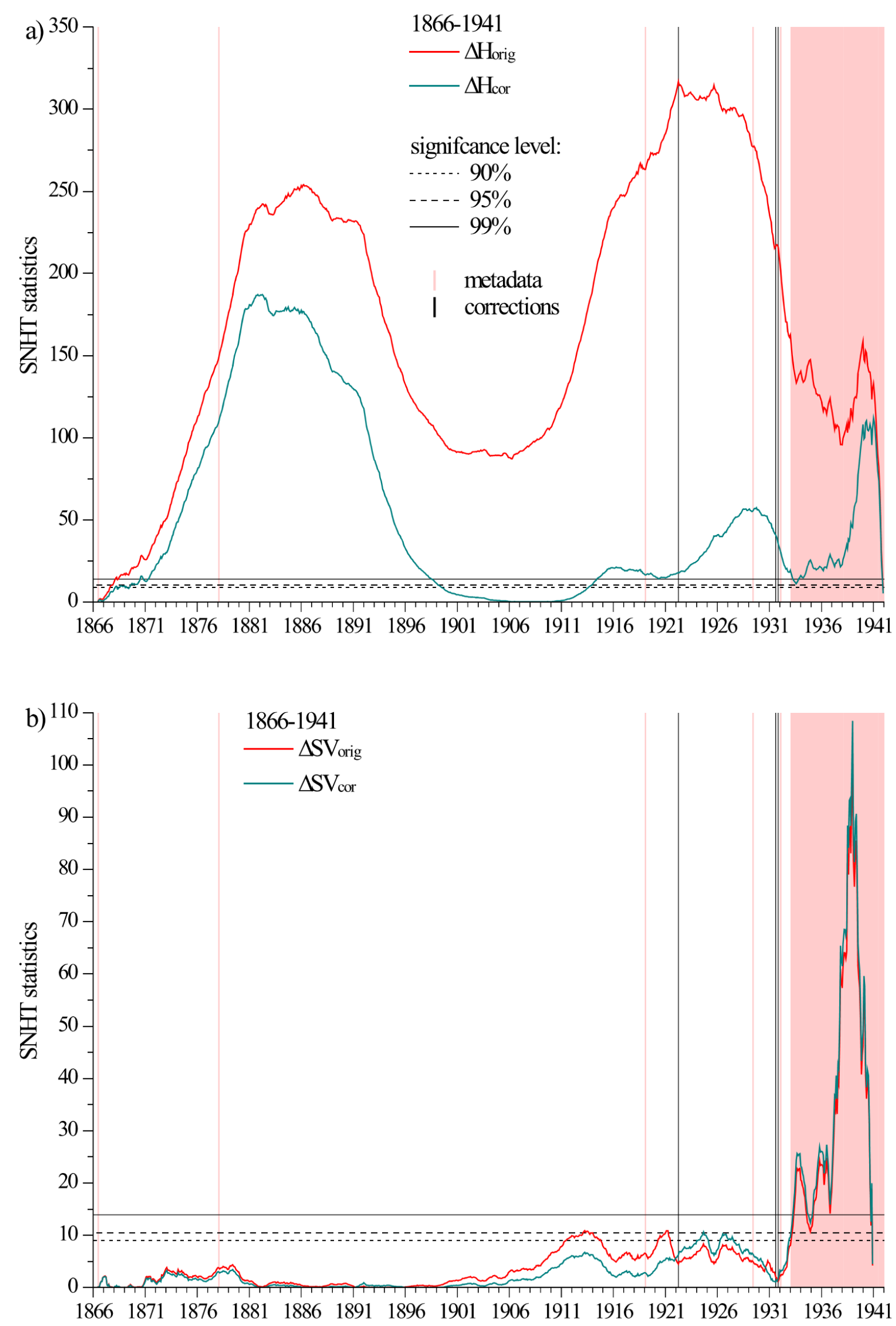

Figure S3. Homogeneity tests statistics for COI: H (a) and SV (b) original (red) and corrected (dark cyan) series for the time interval 1866-1941. Pink vertical lines/rectangles mark possible dates of HB, black vertical lines mark dates of corrected HBs. Dotted, dashed and solid horizontal lines mark levels of $90 \%, 95 \%$ and $99 \%$ of the statistical significance of HBs. 

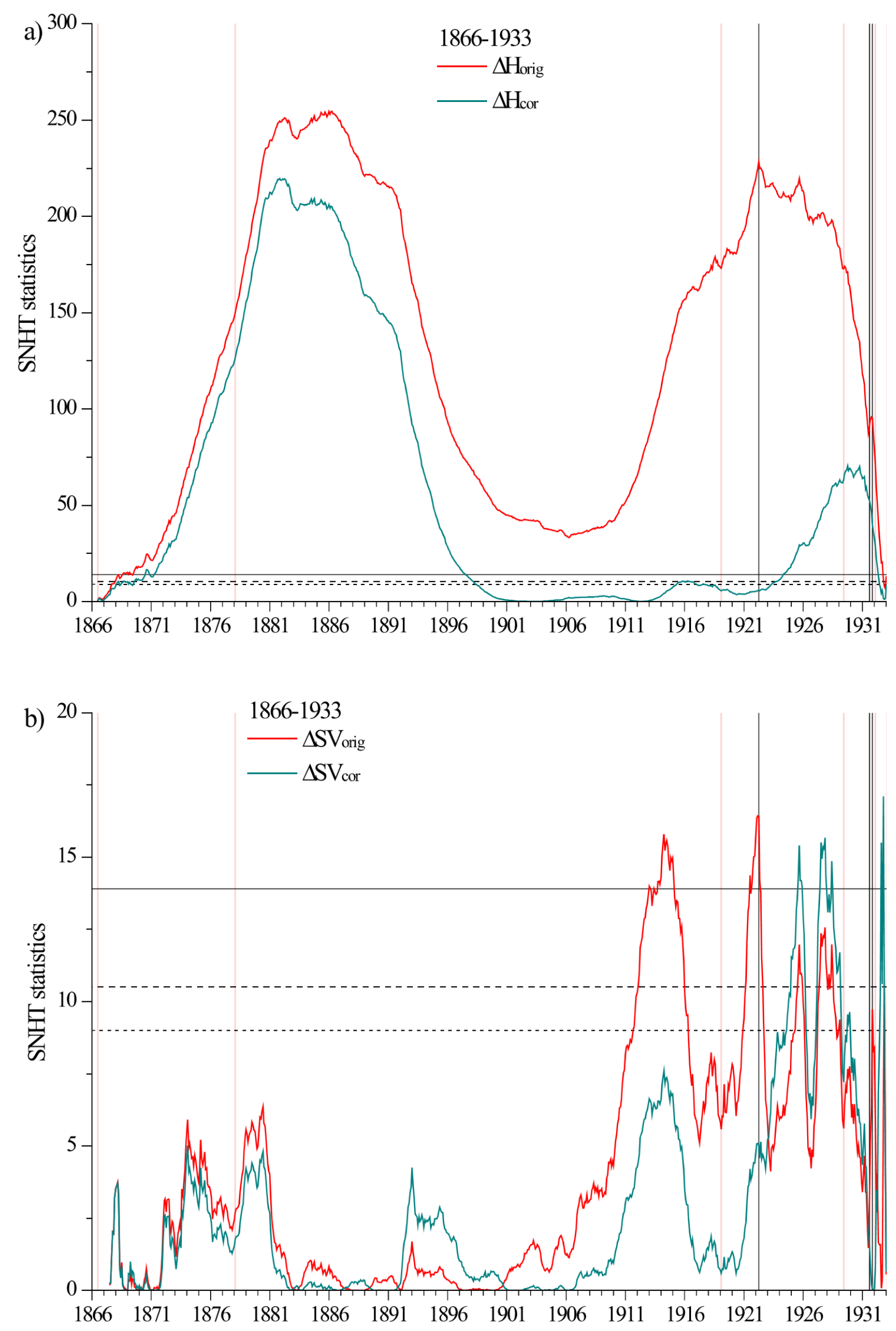

Figure S4. Same as Figure S3 but for the time interval 1866-1933. 

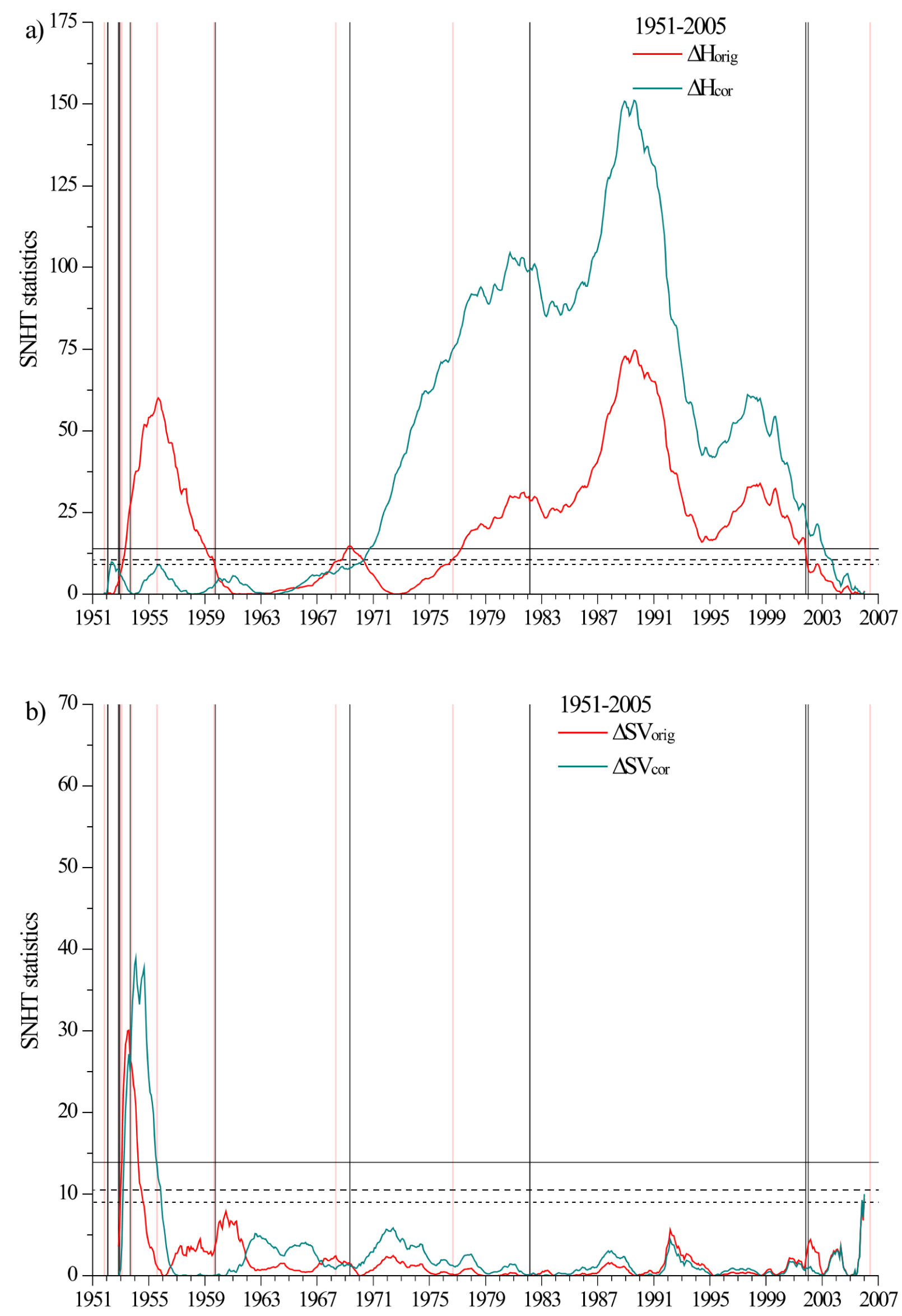

Figure S5. Same as Figure S3 but for the time interval 1951-2005. 

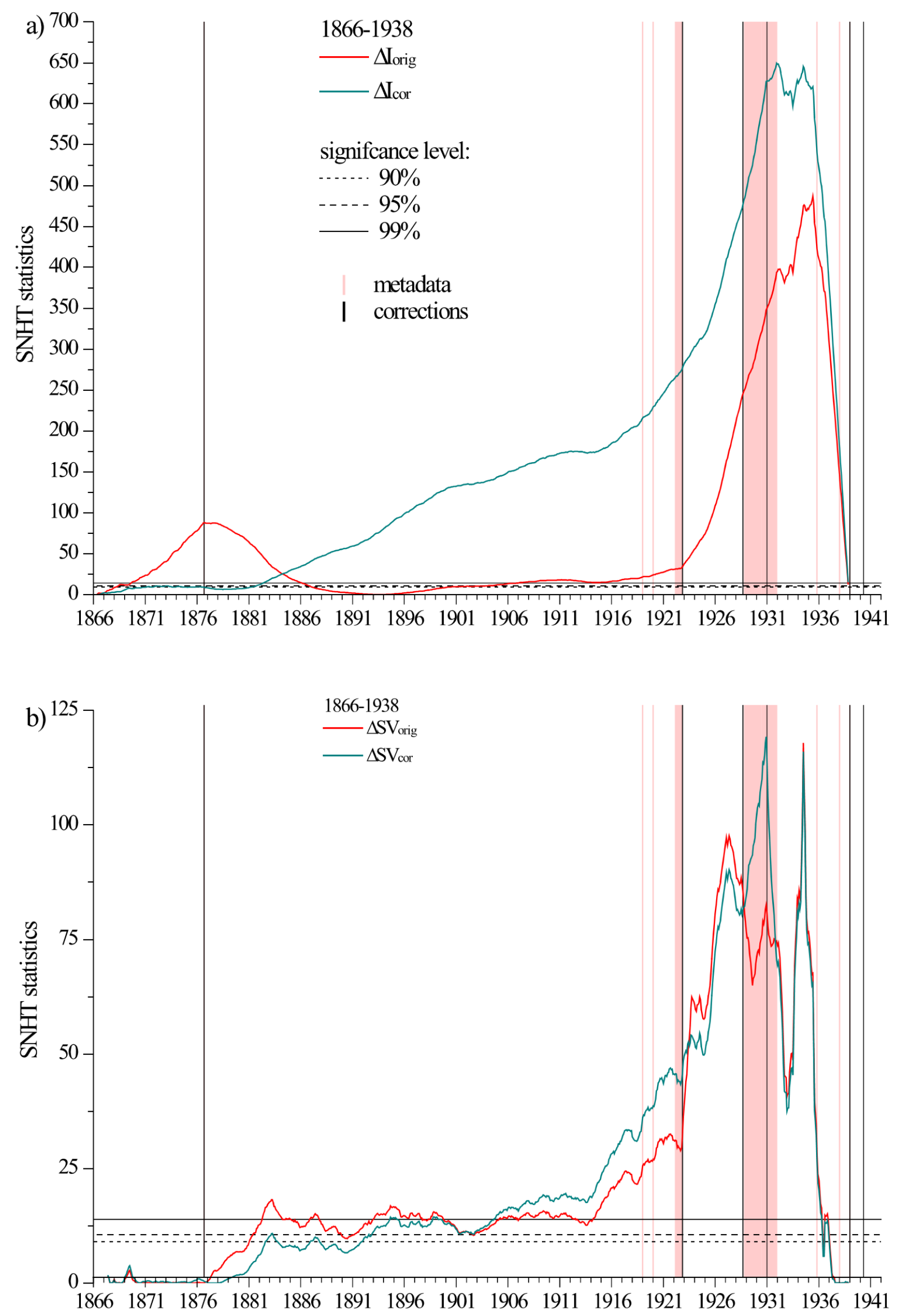

Figure S6. Same as Figure S3 but for COI I. Time interval: 1866-1938. 

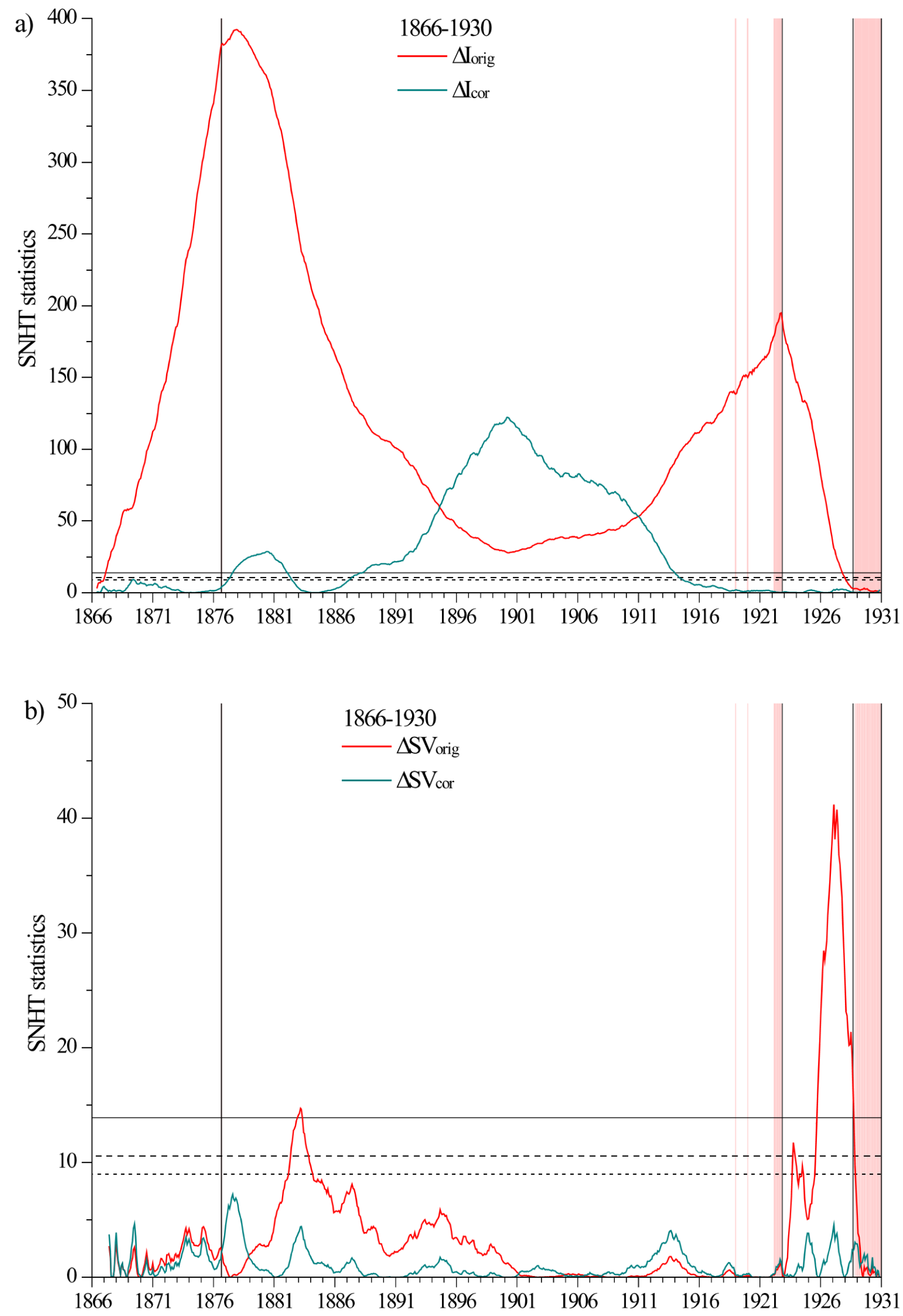

Figure S7. Same as Figure S6 but for the time interval 1866-1930. 

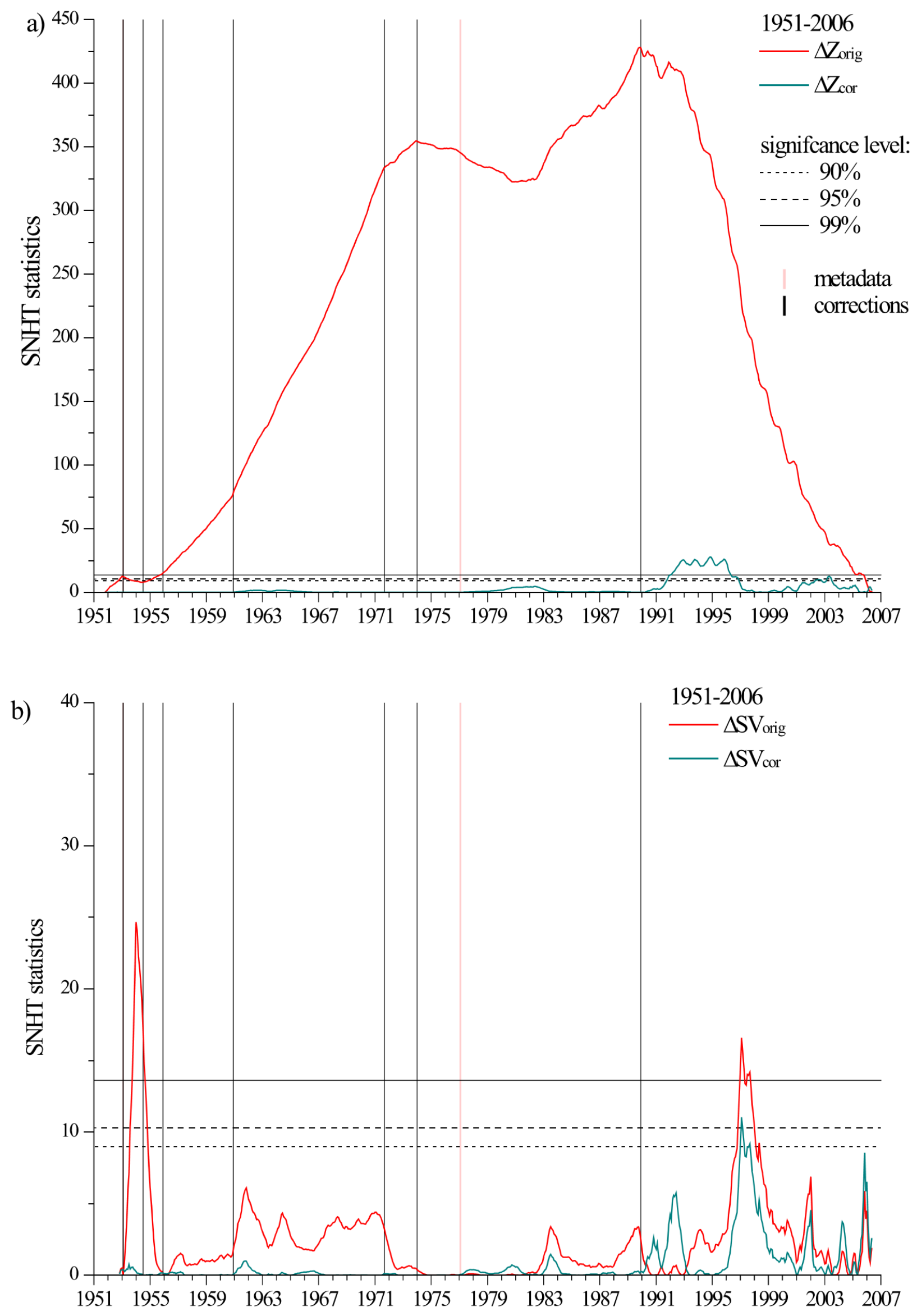

Figure S8. Same as Figure S3 but for COI Z. Time interval: 1951-2006. 

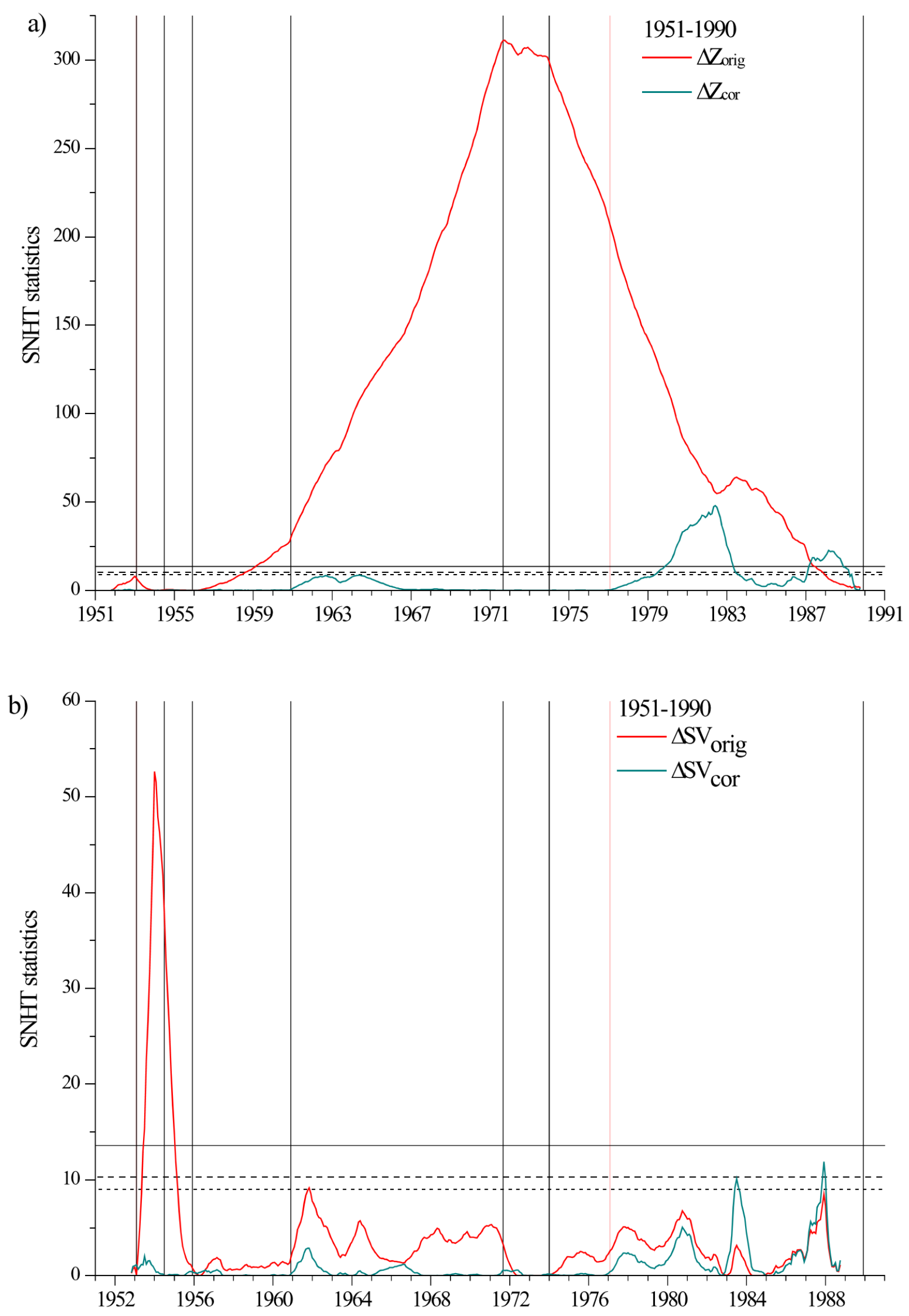

Figure S9. Same as Figure S8 but for the time interval 1951-1990. 

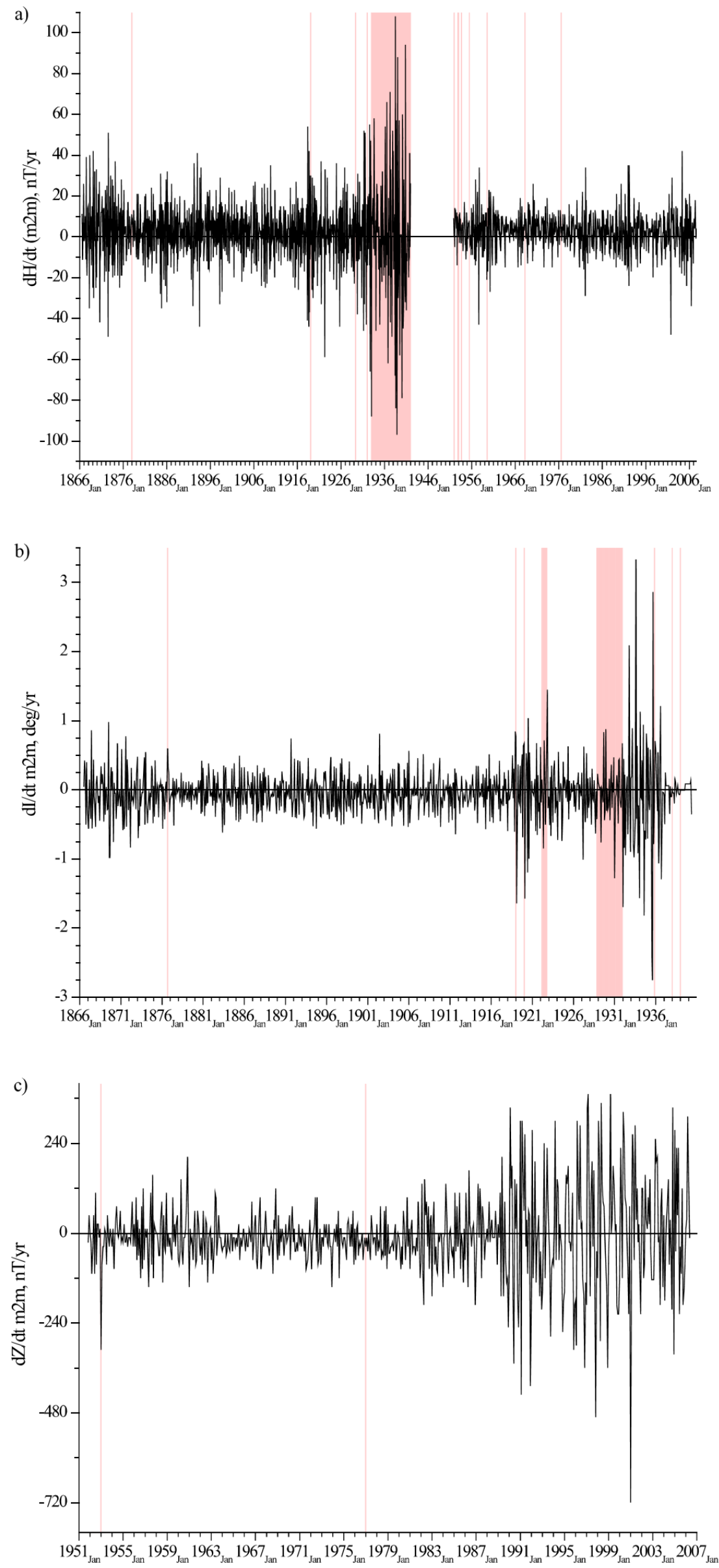

Figure S10. Time derivative of the original COI: H (a), I (b) and Z (c) series on month-to-month time scale. Pink vertical lines/rectangles mark possible dates of HB. 

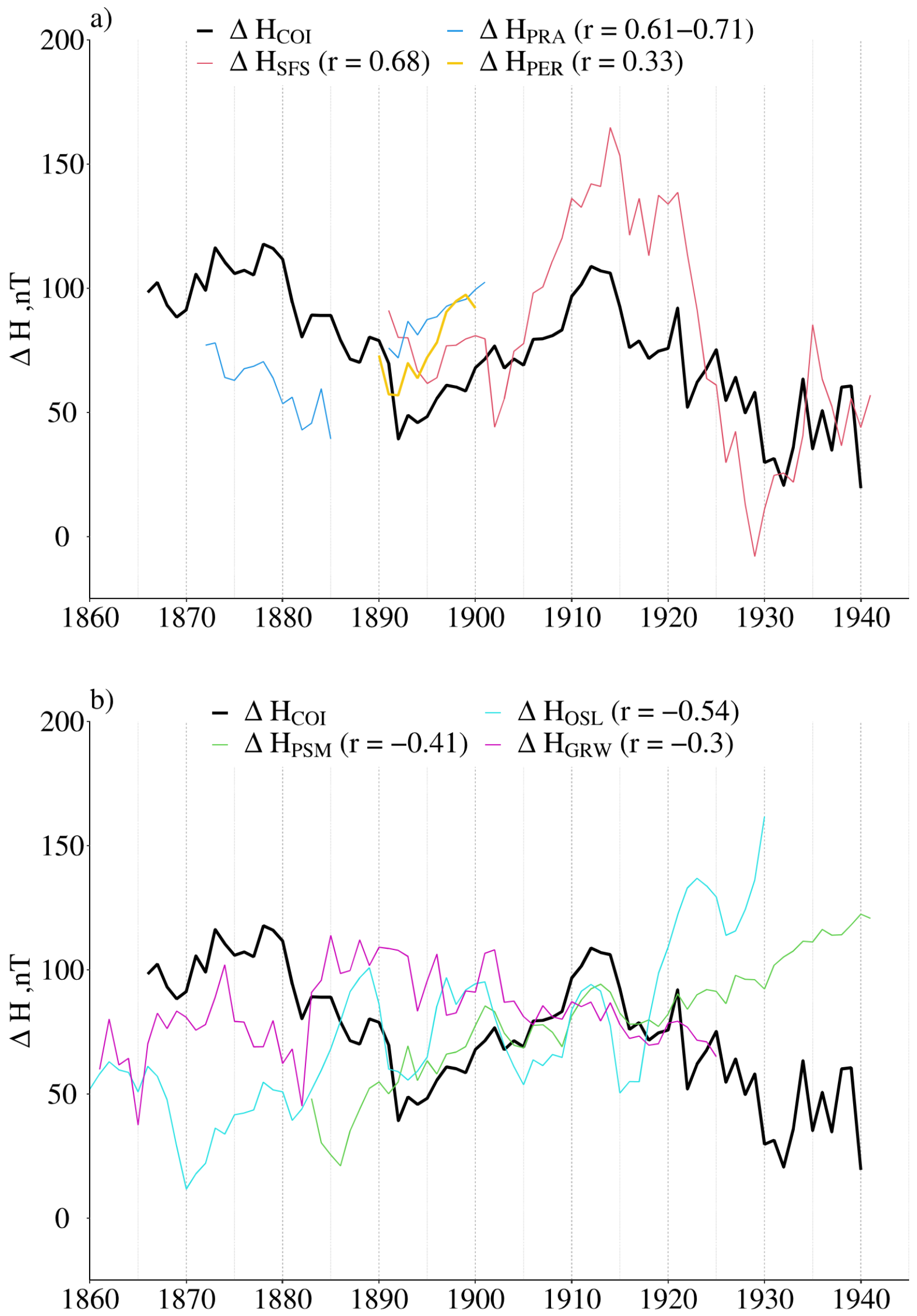

Figure S11. $\triangle H$ series for the 1860-1940 time interval for seven European stations. (a) COI (thick black), SFS (red), PRA (blue) and PER (yellow). (b) COI (thick black), PSM (green), OSL (cyan) and GRW (magenta). 


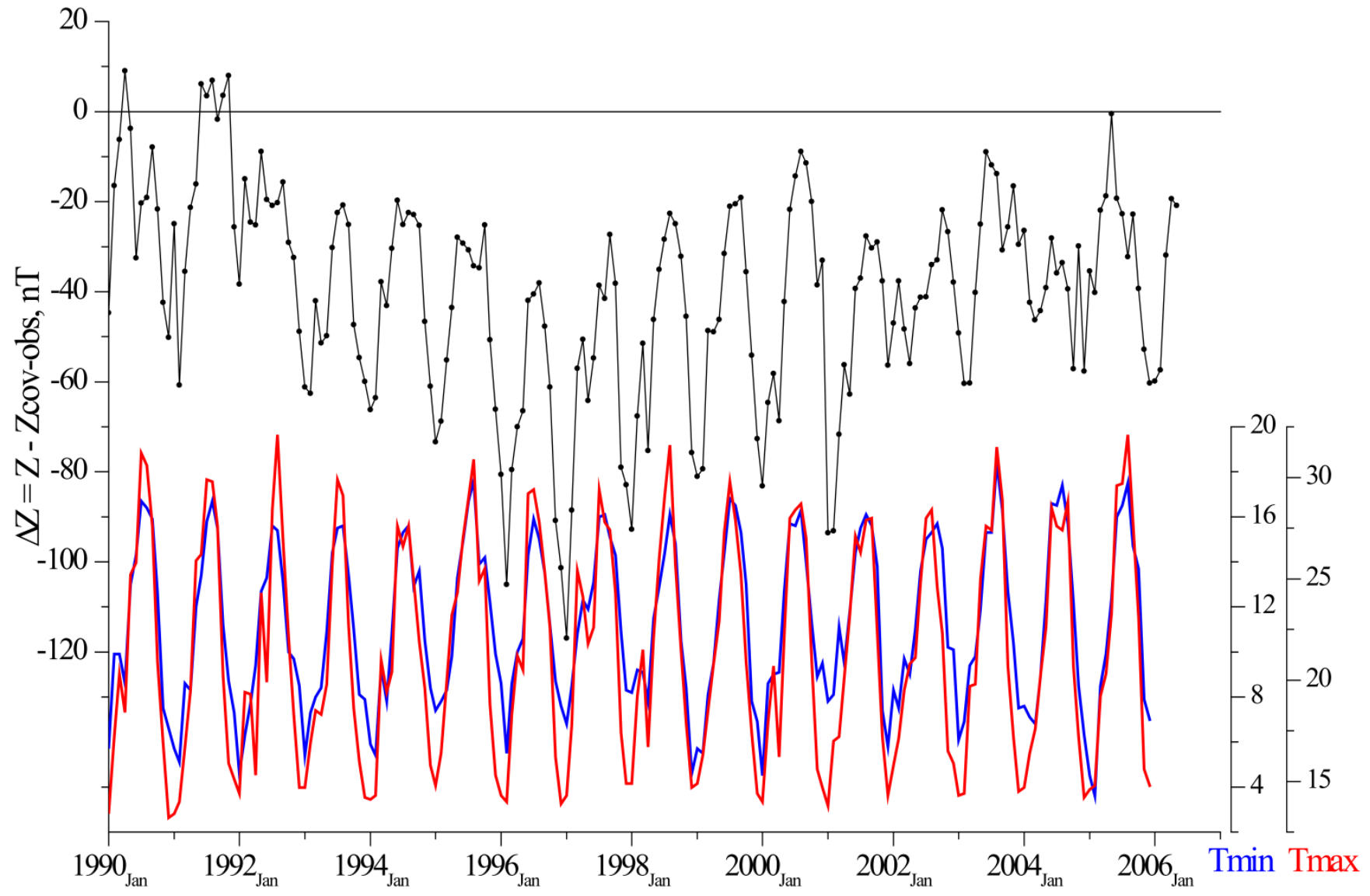

Figure S12. $\Delta$ Z (black line with dots) for the 1990-2006 period together with monthly mean COI temperature series (red - Tmax, blue - Tmin). 